\title{
La formazione a distanza in psicoterapia cognitivo-comportamentale: rischi ed opportunità
}

\author{
Marco Bani*, Michele Procacci**
}

[Ricevuto l'11 dicembre 2020

Accettato il 29 dicembre 2020]

\section{Sommario}

La pandemia da Covid-19 ha modificato radicalmente la pratica clinica, introducendo la necessità di erogare interventi in modalità telematica per molti mesi; al tempo stesso anche le scuole di formazione in psicoterapia hanno dovuto inizialmente sospendere la formazione in presenza per poi riprendere i corsi in modalità telematica, adattando la struttura dei corsi a questa nuova modalità.

La peculiarità della formazione in psicoterapia rende complessa una formazione completamente a distanza soprattutto per la dimensione esperienziale della formazione.

È stata effettuata una SWOT analysis che ha coinvolto i direttori delle scuole di specializzazione in psicoterapia afferenti alla SITCC per identificare i punti di forza e debolezza delle scuole e le opportunità e i rischi che l'attuale situazione determina per la formazione e per gli specializzandi nei prossimi mesi.

I risultati si sono focalizzati su due macro-categorie (opportunità e rischi) che includono alcuni temi e sotto-temi che vengono discussi per fornire alcuni spunti di riflessione e discussione per l'organizzazione di una nuova didattica.

La didattica telematica presenta indubbi vantaggi che tutti i partecipanti hanno sottolineato, tuttavia è importante considerare gli adattamenti necessari ad un uso efficace di questa modalità. La maggior parte delle scuole ha espresso la volontà di mantenere la modalità telematica per una parte della didattica anche in futuro riconoscendone il valore di esperienza formativa.

Parole chiave: formazione in psicoterapia, formazione a distanza, SWOT analysis.

* Ricercatore in Psicologia Clinica, Università di Milano Bicocca.

** Psichiatra, psicoterapeuta cognitivo-comportamentale, didatta SPC, SICC, SITCC, Terzo Centro Psicoterapia Cognitiva. e-mail: procacci@terzocentro.it

Autore corrispondente: Marco Bani, Università di Milano Bicocca, Monza, via Cadore 48, 20092,0264488176. e-mail: marco.bani1@unimib.it

Quaderni di Psicoterapia Cognitiva (ISSN 1127-6347, ISSNe 2281-6046), n. 47/2020

Doi:10.3280/qpc47-2020oa11205 For terms and conditions of usage please see: http://creativecommons.org/. 
Marco Bani, Michele Procacci

\section{Abstract \\ CBT distance training: risks and opportunities}

The Covid-19 pandemic has radically changed clinical practice, introducing the need to deliver interventions electronically for many months; at the same time, psychotherapy training schools also initially had to suspend face-to-face training and then resume courses electronically, adapting the structure of the courses to this new method. The peculiarity of training in psychotherapy makes a completely remote training complex, especially for training's experiential dimension. A SWOT analysis was carried out, involving the directors of the specialization schools in psychotherapy belonging to the SITCC to identify the schools' strengths and weaknesses and the opportunities and risks that the current situation determines for training and trainees in the next months. The results focused on two macro-categories (opportunities and threats), which include some themes and sub-themes discussed to provide some food for thought and discussion for the organization of new teaching. Telematic teaching has undoubted advantages that all the participants underlined. However, it is essential to consider the adaptations necessary for the effective use of this modality. Most of the schools have expressed their desire to maintain the telematic modality for part of the teaching and recognize its value as an educational experience.

Key words: psychotherapy training; distance learning; SWOT analysis.

\section{Introduzione}

La pandemia ha rappresentato una circostanza che ha permesso di mettere in gioco una molteplicità di risorse cliniche e ha moltiplicato le opportunità di intervento, consentendo di fornire interventi in modalità telematica ad un'ampia porzione di popolazione che altrimenti difficilmente avrebbe potuto accedere a risorse psicologiche. Inoltre lo sviluppo di linee guida e indicazioni psicoeducative per la gestione del distress legato al lockdown ha aumentato la consapevolezza del benessere psicologico nella popolazione.

Gli interventi in modalità telematica sono già stati oggetto di attenzione in questi anni, con lo sviluppo di linee guida da parte dell'ordine nazionale degli psicologi e di numerosi studi (Luo et al., 2020; Carlbring et al., 2018); tuttavia sono rimasti essenzialmente interventi secondari, utilizzati solo in casi particolari di difficoltà di accesso alle consultazioni in presenza e mai come prima scelta.

La recente situazione pandemica ne ha invece promosso l'adozione sistematica da parte di tutti gli operatori e in tutti i contesti, con la necessità di formarsi all'uso di strumenti e procedure differenti, adottando le prassi più aggiornate in ambito deontologico e clinico.

Se grande attenzione è stata data all'implementazione degli interventi clinici in modalità telematica, decisamente meno attenzione è stata data alla For terms and conditions of usage please see: http://creativecommons.org/. 
formazione in psicoterapia; la sospensione delle lezioni in presenza ha rappresentato uno stop forzato anche per le scuole di specializzazione (e per migliaia di specializzandi) che hanno dovuto dapprima convertire la didattica in modalità telematica e poi ridefinire la didattica alla luce delle differenti situazioni che si sono di volta in volta determinate.

Formarsi alla psicoterapia comporta, da un lato, l'acquisizione di conoscenze, ma una parte rilevante dei percorsi di formazione comporta attività clinica, simulazioni in coppia o piccolo gruppo, attività esperienziali; le scuole che utilizzano maggiormente la didattica esperienziale si sono quindi scontrate con la necessità di riconvertire questa parte di formazione in modalità telematica o di attendere la ripresa delle attività in presenza, posticipandola nei calendari didattici.

È rilevante in questo contesto domandarsi: è possibile formarsi alla psicoterapia senza attività in presenza? È possibile convertire esperienze di simulazione di colloqui in modalità telematica?

Questa domanda ha attraversato molte professioni (per esempio quella medica o infermieristica) ma essenzialmente a livello pre-laurea, mentre l'attività clinica è stata comunque sempre possibile per i medici in formazione specialistica (pur in condizioni di necessità e con un forte sbilanciamento sul garantire continuità ai servizi sanitari più che sul valore formativo dell'esperienza clinica).

Per gli specializzandi in psicoterapia invece la situazione pandemica ha significato soprattutto la sospensione delle attività di tirocinio e della didattica e una conversione di quest'ultima in forma telematica.

La formazione in psicoterapia in modalità telematica è stata oggetto di diversi studi negli ultimi 10/15 anni, ma è solo recentemente che, grazie anche allo sviluppo tecnologico e degli strumenti telematici, è stato possibile valutare in modo rigoroso l'efficacia di queste modalità.

Inizialmente, i primi lavori che hanno valutato modalità telematiche di formazione alla CBT (Bennett-Levy et al., 2009) partivano dal presupposto che tali programmi erano destinati essenzialmente ad operatori che, per la distanza fisica dai principali centri urbani, non erano in grado di partecipare a formazioni in presenza oppure dovevano sostenere costi eccessivi. Per esempio nel contesto australiano, nelle zone rurali la disseminazione della CBT era problematica soprattutto per questi elementi e alcune indicazioni preliminari suggerivano che un training on-line, affiancato ad una regolare supervisione, poteva ridurre del $50 \%$ il tempo necessario alla formazione in presenza. Tuttavia gli autori sottolineavano che per l'acquisizione di nuove competenze la formazione in presenza era da preferire.

Più recentemente il focus si è spostato fortemente sulla diffusione, con 
corsi telematici, degli interventi basati sulle evidenze nell'area della salute mentale.

Per esempio Jakoby e collaboratori (2019) hanno confrontato corsi di formazione in presenza e on-line per il trattamento del disturbo ossessivocompulsivo, concludendo che entrambi sono efficaci nell'aumentare la conoscenza, competenza e propensione verso l'uso dell'esposizione con prevenzione della risposta da parte dei clinici.

Uno studio recente ha presentato i dati di una revisione sistematica (Frank et al., 2020) dei training focalizzati sugli interventi basati sulle evidenze, che costituiscono una parte rilevante della formazione delle scuole di psicoterapia, pur con accenti differenti. Gli autori hanno incluso 76 studi, di cui 20 in modalità on-line, concludendo che c'è un incremento della conoscenza acquisita e della frequenza di utilizzo degli interventi appresi (benché queste misure di esito siano state raccolte in modalità self report). Inoltre c'è una sostanziale comparabilità con la formazione in presenza in termini di conoscenze e competenze acquisite.

Tra i limiti messi in luce dagli autori c'è però la limitatezza dei follow-up e alcuni risultati contrastanti.

In ambito psichiatrico recentemente è stato sviluppato e implementato un ampio training MOOC (Massive Open On-line Course; Gargot et al., 2020) rivolto a medici psichiatri e focalizzato sulla CBT, che meglio si presta a formazioni specifiche su approcci terapeutici e tecniche di intervento. Gli autori sottolineano come questa modalità rappresenti un'opportunità formativa importante che tuttavia non può sostituire la formazione in presenza e soprattutto presenta il grosso limite della certificazione delle competenze che deve passare necessariamente da momenti di formazione in presenza (role playing, supervisione).

Inoltre, gli autori evidenziano il basso tasso di completamento del modulo MOOC (13\% degli iscritti), tenuto conto della gratuità dell'offerta formativa.

Il ruolo della supervisione si conferma come centrale nella formazione in psicoterapia, soprattutto nel confronto tra formazione in presenza e on-line; se alcuni autori si esprimono negativamente verso la possibilità di una supervisione a distanza e la necessità di prevedere momenti in presenza, altri ritengono invece che il mezzo telematico non costituisca un limite.

Rakovshik et al. (2016) hanno effettuato uno studio randomizzato confrontando una formazione CBT breve con o senza supervisione on-line e un gruppo di controllo, evidenziando come la formazione che includeva la supervisione dava gli esiti migliori in termini di performance clinica valutata da un valutatore esterno. 
La formazione a distanza in psicoterapia cognitivo-comportamentale: rischi ed opportunità

La necessità di affrontare il tema della formazione in modalità telematica anche in ambito nazionale $\mathrm{e} i$ vincoli che la pandemia porrà anche nei prossimi mesi pongono in primo piano una discussione sulla formazione in psicoterapia.

Alla luce di queste considerazioni, il presente lavoro si propone di analizzare l'approccio delle scuole di specializzazione in psicoterapia afferenti alla SITCC alla didattica telematica, con l'obiettivo di mettere in evidenza i principali nuclei tematici e fornire alcune linee guida che possano essere un punto di partenza per una discussione più sistematica.

\section{Metodo}

Per indagare l'impatto della pandemia da Covid-19 sulla formazione in psicoterapia è stata condotta una SWOT analysis che ha coinvolto i direttori delle scuole di psicoterapia affiliate alla SITCC. L'analisi si è focalizzata in particolare sui fattori facilitanti e le barriere all'implementazione efficace della didattica a distanza/telematica nella formazione in psicoterapia.

L'acronimo SWOT racchiude quattro aree: Punti di forza (Strengts), Punti di debolezza (Weaknesses), Opportunità (Opportunities) e Rischi (Threats) e descrive un approccio che è stato utilizzato primariamente nel contesto aziendale, per analizzare i principali fattori che impattano sulle performance aziendali o lo sviluppo di nuove iniziative, tuttavia è stato implementato successivamente in molteplici contesti in cui fosse necessario identificare i fattori che contribuiscono a guidare le scelte organizzative in uno scenario futuro (Leong et al., 2007; Uhrenfeldt et al., 2014).

Sulla base degli elementi individuati dagli stakeholders di un'organizzazione è possibile ipotizzare una strategia che sfrutti le opportunità delle organizzazioni (nel nostro caso le scuole di formazione) facendo leva sui loro punti di forza, affrontando in modo proattivo le minacce e correggendo o compensando i punti deboli.

Un'analisi SWOT utilizza domande aperte semi-strutturate per identificare aspetti favorevoli e sfavorevoli in quattro quadranti. I punti di forza (S) e di debolezza $(\mathrm{W})$ costituiscono i fattori interni, mentre le opportunità $(\mathrm{O})$ e le minacce $(\mathrm{T})$ sono fattori esterni.

\section{Partecipanti e raccolta dei dati}

I dati sono stati raccolti nell'estate 2020 (tra giugno e agosto) Sono stati contattati via mail i direttori delle 40 scuole affiliate alla SITCC; di queste 
24 hanno declinato l'invito o non hanno risposto (anche dopo un secondo invito di promemoria). Per facilitare la partecipazione, in ragione delle circostanze e delle limitazioni imposte dalla pandemia, è stata data la possibilità ai direttori di essere contattati per un'intervista telefonica o telematica oppure di inviare in forma scritta (come documento di testo) il proprio contributo. 3 hanno scelto l'intervista in modalità telematica e 5 hanno scelto l'invio di un contributo scritto. Complessivamente sono state incluse nell'analisi le narrative di 8 direttori, in rappresentanza di 16 scuole $(40 \%$ delle scuole affiliate).

Le domande semistrutturate proposte ai partecipanti sono state le seguenti:

- quali sono i punti di forza e i punti di debolezza delle scuole di formazione in psicoterapia, facendo particolare riferimento alle vostre scuole (al netto della situazione Covid-19);

- alla luce dell'attuale situazione quali sono le opportunità che vedete per la formazione? Quali possibilità si aprono che prima erano precluse o non considerate? Quali opportunità hanno i docenti delle scuole e quali gli specializzandi?

- alla luce dell'attuale situazione quali sono invece i rischi che intravedete? quali le possibili conseguenze negative per le scuole e per gli specializzandi?

La SWOT analysis è stata utilizzata per mettere in luce le quattro categorie tematiche che la caratterizzano - i punti di forza (Strenghts), le debolezze (Weaknesses), le opportunità (Opportunities) e i rischi (Threats) - applicandola al contesto della formazione in psicoterapia. Quindi ai partecipanti è stato chiesto di esprimere il proprio parere sulle quattro categorie tematiche facendo riferimento alle proprie scuole di formazione, alle modalità con cui è stata portata avanti la formazione nei mesi del lockdown e della successiva parziale riapertura e alla pianificazione dei prossimi mesi.

Le due modalità di raccolta dei contributi (documento di testo $v s$ intervista) hanno determinato un differente livello di approfondimento, tuttavia si è ritenuto di favorire il maggior coinvolgimento a scapito dell'omogeneità delle narrative.

\section{Analisi dei dati}

Uno degli autori (MB) ha letto tutti i contributi e ha effettuato un'analisi di contenuto (content analysis, Graneheim e Lundman, 2004) seguen- 
do cinque passaggi: identificazione delle unità di significato; formulazione delle unità di significato in descrizioni aderenti al testo; unità interpretative dei significati sottesi; identificazione dei sotto-temi; presentazione dei temi.

Quindi sono stati identificati i nuclei tematici riportati dai direttori, organizzandoli in categorie tematiche affini. Il secondo autore (MP) ha poi rivalutato le categorie proposte applicandole post hoc ai contributi testuali per verificarne la capacità esplicativa; inoltre, i temi individuati dal primo autore sono stati discussi fino al raggiungimento di un consenso.

In tabella 1 sono presentate le categorie tematiche emerse e i relativi temi e sotto-temi sviluppati in ogni categoria.

Tab. 1 -SWOT analysis: categorie, temi e sotto-temi

\begin{tabular}{|c|c|c|}
\hline Categorie & Temi & Sotto-temi \\
\hline \multirow[t]{3}{*}{ Opportunità } & Occasione formativa & $\begin{array}{l}\text { Conoscenza dinamiche } \\
\text { personali } \\
\text { Crescita competenza didattica }\end{array}$ \\
\hline & Risparmio/efficienza & $\begin{array}{l}\text { Risparmio di tempo per il } \\
\text { docente/per lo studente } \\
\text { Risparmio economico per la } \\
\text { scuola/lo studente } \\
\text { Rispetto dei tempi didattici e } \\
\text { delle pause }\end{array}$ \\
\hline & Accessibilità & $\begin{array}{l}\text { Maggiore accesso per } \\
\text { studenti } \\
\text { Maggiore accesso per docenti } \\
\text { Archiviazione materiale }\end{array}$ \\
\hline \multirow[t]{5}{*}{ Rischi } & Didattica esperienziale & $\begin{array}{l}\text { Difficoltà di implementare il } \\
\text { training formativo } \\
\text { esperienziale }\end{array}$ \\
\hline & Attenzione/partecipazione & $\begin{array}{l}\text { Difficoltà tecniche } \\
\text { Difficoltà di attenzione } \\
\text { prolungata } \\
\text { Difficoltà di monitoraggio } \\
\text { dell'esperienza }\end{array}$ \\
\hline & Socialità & $\begin{array}{l}\text { Perdita di occasioni informali } \\
\text { di confronto } \\
\text { Costruzione del gruppo }\end{array}$ \\
\hline & Setting, etica & \\
\hline & Scuole minori & Meno risorse \\
\hline
\end{tabular}




\section{Risultati}

Le risposte inviate dai direttori delle scuole che hanno accettato di partecipare allo studio sono state codificate attorno a due macrocategorie: opportunità e rischi.

Le aree di punti forza e debolezza delle scuole sono state affrontate in modo chiaro solo da due dei partecipanti, si è quindi ritenuto maggiormente funzionale mantenere il focus sulle due macrocategorie indicate in tabella 1.

\section{Opportunità}

All'interno della prima macrocategoria sono stati individuati 3 temi: occasione formativa, risparmio/efficienza, accessibilità.

Il primo tema, l'occasione formativa, ha messo in luce il valore formativo della situazione pandemica, utilizzandola come un'opportunità per sviluppare la competenza didattica, cioè la necessità di acquisire le conoscenze e le competenze per svolgere didattica telematica ("Il primo vantaggio per noi come Scuola... è stato quello di doversi dotare di nuove categorie di lettura della realtà didattica, di modalità poco praticate"). Da un lato viene evidenziato l'aspetto tecnico-didattico e cioè la scelta dello strumento più adatto alle caratteristiche della didattica che si vuole erogare in funzione delle sue possibilità di interazione (es. la possibilità di attività in piccolo gruppo, di registrazione), dall'altro l'aspetto più pedagogico declinato o sul versante dell'adattamento della didattica al nuovo strumento o alla costruzione di una nuova didattica che non si limiti a riprodurre le modalità precedenti ("Non è che noi stiamo facendo una cosa un po' meno bella, stiamo facendo un'altra cosa; cioè non stiamo mangiando una pizza meno buona, stiamo mangiando un risotto alla parmigiana ... il confronto sarebbe stato devastante...").

Un ulteriore sotto-tema è la possibilità di usare la situazione pandemica e la didattica telematica come occasione di conoscenza personale per gli specializzandi (oltre che per i docenti stessi), su cui lavorare anche nelle lezioni esperienziali ("Vi incoraggio a riflettere su come stiate vivendo questo momento, accorgendoci tutti insieme che il problema non è il virus, ma quello che attiva dentro di noi. Se possiamo stare meglio è affrontando $i$ nostri nodi, l'epidemia è solo l'ennesima occasione per farlo").

Un secondo tema, l'efficienza, è trasversale a tutte le scuole ed è stato declinato sia sul versante temporale che economico; l'introduzione della didattica a distanza ha comportato un maggiore rispetto dei tempi didattici, con un inizio più puntuale delle lezioni di training e un maggiore rispetto 
delle pause didattiche ("Dal punto di vista del setting formativo, banalmente la FAD costringe alla puntualità docenti e allievi ed è quindi molto educativa per quegli allievi e per quei docenti usualmente un po' lassi dal punto di vista delle regole di lavoro").

L'efficienza è stata poi ampliata al tema del risparmio di tempo e di risorse economiche; le scuole hanno sottolineato come vantaggio evidente la riduzione dei tempi di spostamento per i docenti e per gli studenti e un risparmio economico diretto legato ai costi logistici.

Al tempo stesso la maggiore efficienza e le risorse economiche hanno consentito un investimento negli strumenti necessari a fornire una didattica a distanza di qualità tecnica adeguata e in prospettiva molte scuole hanno segnalato l'opportunità di costruire occasioni formative con contributi di clinici e ricercatori nazionali e internazionali che in precedenza non avrebbero potuto essere contattati (“... poter invitare docenti internazionali abbattendo drammaticamente i costi per la scuola. La possibilità di allargare il bouquet di docenti rende possibile agli allievi di oggi una possibilità di apprendere da docenti da tutto il mondo e di poterli conoscerli e fare network").

Un terzo tema è quello dell'accessibilità, infatti le scuole hanno sottolineato la possibilità di registrazione delle lezioni telematiche, consentendo da un lato la possibilità di recupero delle lezioni o di parte dei contenuti (almeno quelli più frontali e meno esperienziali) in caso di assenza, dall'altro la creazione di un bagaglio di video didattici accessibile e utilizzabile in diversi contesti formativi e più flessibile rispetto al calendario didattico standard ("Altro grande vantaggio è quello di poter facilmente videoregistrare le lezioni in maniera semplice e immediatamente disponibile per tutti gli allievi. Un patrimonio a disposizione degli allievi'). Infine, tutte le scuole hanno sottolineato la possibilità di ampliare l'offerta didattica con nuovi docenti nazionali/ internazionali che sarebbero stati inaccessibili in precedenza per questioni logistiche ed economiche e invece in modalità telematica sono più facilmente contattabili e disponibili anche a fronte di investimenti più limitati.

\section{Rischi}

All'interno della seconda macrocategoria sono stati individuati 5 temi: didattica esperienziale, attenzione/partecipazione, socialità, etica/setting, dimensioni scuole.

Il principale rischio, raccolto nel primo tema, e sottolineato da quasi tutti i partecipanti, è relativo alla didattica esperienziale, in particolare la difficoltà ad implementarla in modalità telematica, difficoltà che talvolta assume la forma della impossibilità. 
Il valore dell'interazione in presenza viene riconosciuto come non sostituibile ("Si perde globalmente la corporeità dell'altro, la possibilità che viene dall'utilizzo dei registri prossimali, la possibilità di costruire, regolare e mantenere quel clima emotivo necessario al corretto andamento di una esercitazione/seduta, la possibilità di calibrare in modo pieno la dimensione senso-motoria e tutte le sue inevitabili fluttuazioni, la possibilità di sperimentare il contatto e lo scambio diretto col docente").

Legato a questo tema viene poi segnalato il rischio di una maggiore difficoltà di monitorare lo sviluppo dell'identità professionale degli specializzandi e di riconoscere eventuali momenti di difficoltà ("La formazione a distanza inoltre impedisce di cogliere sfumature comportamentali degli allievi, importanti per i trainer al fine della scoperta di punti di debolezza di questi ultimi”).

Un secondo tema nell'area dei rischi si focalizza sugli aspetti tecnici e cognitivi della didattica in modalità telematica; da un lato viene posta l'attenzione sulle difficoltà tecniche nell'approccio alla didattica telematica, in particolare la mancanza di competenze soprattutto da parte di alcuni docenti che hanno maggiori difficoltà di adattamento alla modalità telematica. In secondo luogo, viene sottolineato l'impatto negativo in termini di attenzione sostenuta da parte degli studenti e la ridotta partecipazione ("Attraverso la formazione on-line gli studenti tendono più facilmente a distrarsi e sono meno partecipativi"). La giornata di formazione piena (8 ore) risulta troppo faticosa in modalità telematica sia per i docenti che per gli studenti.

Inoltre, la maggiore difficoltà nel raccogliere feedback non verbali durante la lezione limita le occasioni di partecipazione da parte degli studenti.

Infine, un terzo tema di rischio è relativo all'impatto negativo della didattica telematica sulla socializzazione tra gli studenti intesa sia come occasione di costruzione di legami di gruppo formativo funzionali alle attività di simulazione/lavoro personale che richiedono un significativo livello di fiducia reciproco, sia come perdita di occasioni di incontro informale per approfondire tematiche didattiche, o più semplicemente costruire e condividere amicizie. Ciò in particolare per i gruppi di specializzandi che si sono formati da meno tempo e che hanno avuto quindi meno opportunità di creare legami solidi e questo aspetto porta con sé la preoccupazione di una minore fiducia di gruppo e una ridota disponibilità all'apertura durante le attività esperienziali che comportano una apertura su aspetti personali (" $\mathrm{La}$ formazione a distanza limita le possibilità di interazione tra gli allievi e dunque la creazione di un clima di fiducia e sicurezza potendo ciò incidere negativamente sulla motivazione degli stessi ad esporsi per le esercitazioni e le simulazioni durante $i$ training"). 
Un quarto tema si sviluppa attorno al setting e alla dimensione etica; in particolare viene sottolineato come la definizione di nuove regole del setting formativo sia necessaria per entrare nella nuova modalità didattica, analogamente a quanto accade con il setting clinico ("Il setting di lavoro può diventare un grave problema laddove l'allievo (ma talvolta anche il docente) non possa, o piuttosto non sia cosi attento sul piano personale a mantenere un contesto di adeguata riservatezza, sufficientemente rispettoso del lavoro di gruppo"). Inoltre il setting telematico, in mancanza di una riflessione metodologica, espone al rischio di collusività (" $\grave{E}$ stato anche interessante notare la possibile collusività tra alcuni aspetti insiti nelle modalità FAD e la struttura di personalità di alcuni docenti/allievi") ed è quindi importante utilizzarlo non solo come semplice strumento di erogazione didattica ma come contesto formativo di per sé, in cui far emergere proprie caratteristiche di funzionamento su cui lavorare analogamente ad altri contenuti.

Un quinto tema nella categoria dei rischi, portato da alcuni direttori, è relativo all'impatto della didattica a distanza sulle scuole di formazione più piccole o di più recente formazione che, pur potendo avvalersi degli stessi strumenti tecnici di altre scuole, riportano come maggiormente critica la ridotta possibilità di interazione in presenza (che un gruppo piccolo per sua natura permette e che la didattica a distanza tende a togliere). Inoltre, la cancellazione e l'impossibilità di open day in presenza limita fortemente la possibilità di promozione delle scuole più giovani a vantaggio di quelle più consolidate.

\section{Discussione}

Le tematiche che i direttori delle scuole hanno affrontato hanno evidenziato un duplice registro di approccio alla didattica in modalità telematica, con un riconoscimento delle opportunità che si aprono anche in prospettiva futura (al di là delle necessità contingenti legate alla pandemia) e una consapevolezza dei limiti di questa modalità.

L'analisi SWOT presenta dei chiari limiti che è importante esplicitare per una lettura critica delle indicazioni fornite; in primo luogo, è un approccio che favorisce la brevità alla profondità delle risposte, benché la scelta di proporre ai partecipanti una compilazione in forma scritta o un'intervista, abbia in parte compensato questa limitazione fornendo però contenuti disomogenei per livello di profondità tematica.

Un secondo aspetto è legato alla natura auto riferita delle opinioni dei partecipanti, senza un riscontro analitico, pur considerando che gli sta- 
keholders coinvolti hanno un ruolo e un'esperienza particolarmente ampia. Certamente l'uso di approcci metodologici differenti può costituire un passaggio ulteriore per confermare, completare o modificare le indicazioni emerse dal lavoro; per esempio, l'uso di interviste qualitative con docenti e specializzandi sull'esperienza didattica a distanza. Inoltre, l'integrazione della prospettiva degli specializzandi consentirebbe un quadro più completo.

Tuttavia, il lavoro costituisce uno dei primi contributi utili ad una riflessione condivisa sull'adattamento della formazione in psicoterapia alla didattica a distanza, durante e dopo la situazione contingente alla pandemia Covid-19.

Le scuole hanno complessivamente saputo affrontare la situazione di criticità e stanno riuscendo a costruire opportunità formative pur con gradi differenti di difficoltà.

Restano aperte delle aree rilevanti che non sono state discusse in questo lavoro ma che rivestono un ruolo centrale nella formazione, in particolare le attività di tirocinio professionalizzante che costituiscono un'ampia parte del percorso formativo e su cui al momento non ci sono esperienze telematiche analoghe a quelle già provate in ambito sanitario (Lucei et al., 2020; Lee et al., 2020).

\section{Aspetti da considerare per una nuova didattica nelle Scuole di Psicote- rapia in modalità telematica}

Sulla base delle tematiche emerse dai contributi delle scuole è possibile fornire alcuni suggerimenti che possono servire da spunto per lo sviluppo di una discussione più ampia sulla didattica a distanza.

1. Integrazione tra didattica in presenza e a distanza: la didattica a distanza non sostituisce tutta la formazione ma ne costituisce un complemento importante sia dal punto di vista delle opportunità che da quello più strettamente clinico (replicando un setting che gli specializzandi sperimenteranno con maggiore frequenza anche nell'attività clinica).

2. Etica della didattica a distanza: è importante concordare/definire le regole del setting telematico per gestire al meglio le situazioni che possono interferire con l'esperienza formativa; allo stesso modo è importante prevedere una formazione all'etica dell'applicazione clinica del setting telematico.

3. Non un semplice strumento ma un contesto: è opportuno evitare di usare la didattica a distanza solo come un mezzo (fare la stessa cosa ma in modalità telematica) ma come una nuova dimensione formativa sia per $\mathrm{i}$ 
formatori che per gli specializzandi; questo comporta l'adattamento sia della didattica frontale che di quella esperienziale alla modalità telematica, pur consapevoli che la didattica esperienziale richiede anche attività in presenza.

4. Possibilità di differenziare le forme di insegnamento in base all'utilizzo della didattica in presenza (ad esempio, il training di base) rispetto a lezioni a carattere accademico (ad esempio giornate di formazione su temi generali del cognitivismo) che potrebbero essere on-line e registrate. Oppure dividere la stessa materia, ad esempio i disturbi di personalità, in moduli on-line e lezioni in presenza con discussione di casi clinici), ecc.

\section{Conclusioni}

La situazione pandemica che ha segnato profondamente il 2020 ha avuto effetti rilevanti anche sulla formazione in psicoterapia; le scuole hanno saputo reagire in modo efficace, proponendo una didattica telematica che tenga conto delle esigenze specifiche della formazione specialistica.

Indipendentemente dalla situazione sanitaria, le scuole hanno espresso la volontà di mantenere una parte della didattica in modalità telematica, apprezzandone alcuni vantaggi e riconoscendone alcuni limiti. La riflessione è solo all'inizio e c'è spazio per lo sviluppo di una formazione più efficace e più efficiente che si accompagni allo sviluppo di un'area di ricerca che ne valuti l'implementazione.

\section{Ringraziamenti}

Gli autori ringraziano le scuole che hanno gentilmente dato la disponibilità a partecipare allo studio:

SPC - Scuola di psicoterapia Cognitiva;

APC - Associazione di Psicologia Cognitiva;

SBPC - Scuola Bolognese di Psicoterapia Cognitiva;

ATC - Associazione di Terapia Cognitiva;

CTC - Centro Terapia Cognitiva;

IGB - Istituto Gabriele Buccola;

CBT Academy;

CTCC - Scuola di Psicoterapia Cognitivo-Comportamentale. 


\section{BIBLIOGRAFIA}

Bennett-Levy J., Hawkins R., Perry H., Cromarty, P., Mills J. (2012). On-line cognitive behavioural therapy training for therapists: Outcomes, acceptability, and impact of support. Australian Psychologist, 47(3): 174-182. DOI: 10.1111/j.17429544.2012.00089.x

Bennett-Levy J., McManus F., Westling B.E., Fennell M. (2009). Acquiring and refining CBT skills and competencies: which training methods are perceived to be most effective? Behavioural and Cognitive Psychotherapy, 37(5): 571-583. DOI: $10.1017 / \mathrm{s} 1352465809990270$

Calder R., Ainscough T., Kimergård A., Witton J., Dyer K.R. (2017). On-line training for substance misuse workers: A systematic review. Drugs: Education, Prevention and Policy, 24(6): 430-442. DOI: 10.1080/09687637. 2017.1318113

Carlbring P., Andersson G., Cuijpers P., Riper H., Hedman-Lagerlöf E. (2018). Internet-based vs. face-to-face cognitive behavior therapy for psychiatric and somatic disorders: An updated systematic review and meta-analysis. Cognitive Behaviour Therapy, 47(1): 1-18. DOI: 10.1080/16506073.2017.1401115

Frank H.E., Becker-Haimes E.M., Kendall P.C. (2020). Therapist training in evidence-based interventions for mental health: A systematic review of training approaches and outcomes. Clinical Psychology and Science Practice, 27(3): e12330. DOI: $10.1111 /$ cpsp. 12330

Gargot T., Arnaoutoglou N.A., Costa T., Sidorova O., Liu-Thwaites N., Moorey S., Hanon C. (2020). Can we really teach cognitive behavioral therapy with a massive open on-line course? European Psychiatry, 63(1): e38, 1-3. DOI: 10.1192/j. eurpsy.2020.29

Graneheim U.H., Lundman B. (2004) Qualitative content analysis in nursing research: concepts, procedures and measures to achieve trustworthiness. Nurse Education Today, 24: 105-112. DOI: 10.1016/j.nedt.2003.10.001

Jackson C.B., Quetsch L.B., Brabson L.A., Herschell A.D. (2018). Web-based training methods for behavioral health providers: A systematic review. Administration and Policy in Mental Health and Mental Health Services Research, 45(4): 587-610. DOI: 10.1007/s10488-018-0847-0

Lee J., Kim H., Kim K.H., Jung D., Jowsey T., Webster C.S. (2020). Effective virtual patient simulators for medical communication training: A systematic review. Med Educ., 54: 786-795. DOI: 10.1111/medu.14152

Leong F.T.L., Leach M.M. (2007). Internationalising Counseling Psychology in the United States: A SWOT Analysis. Applied Psychology: An International Review, 56(1): 165-181. DOI: 10.1111/j.1464-0597.2007.00283.x

Lucey C.R., Johnston S.C. (2020). The Transformational Effects of Covid-19 on Medical Education. JAMA, 324(11): 1033-1034. DOI: 10.1001/jama.2020.14136

Luo C., Sanger N., Singhal N., Pattrick K., Shams I., Shahid H., Hoang P., Schmidt J., Lee J., Haber S., Puckering M., Buchanan N., Lee P., Ng K., Sun S., Kheyson S., Chung D.C., Sanger S., Thabane L., Samaan Z. (2020). A comparison of electronically-delivered and face to face cognitive behavioural therapies in de-

60

Copyright (c) FrancoAngeli

This work is released under Creative Commons Attribution - Non-Commercial - NoDerivatives License. For terms and conditions of usage please see: http://creativecommons.org/. 
La formazione a distanza in psicoterapia cognitivo-comportamentale: rischi ed opportunità

pressive disorders: A systematic review and meta-analysis. EClinicalMedicine, 24: 100442. DOI: 10.1016/j.eclinm.2020.100442

Rakovshik S.G., McManus F., Vazquez-ontes M., Muse K., Ougrin D. (2016). "Is Supervision Necessary?". Examining the Effects of Internet-Based CBT Training With and Without Supervision. Journal of Consulting and Clinical Psychology, 84(3): 191-199. DOI: 10.1037/ccp0000079)

Uhrenfeldt L., Lakanmaa R.-L., Flinkman M., Basto M.L., Attree M. (2014). Collaboration: a SWOT analysis of the process of conducting a review of nursing workforce policies in five European countries. Journal of Nursing Management, 22: 485-498. DOI: 10.1111/j.1365-2834.2012.01466.x For terms and conditions of usage please see: http://creativecommons.org/. 